\title{
Regional variations in fluoroquinolone non- susceptibility among Escherichia coli bloodstream infections within the Veterans Healthcare Administration
}

Daniel J. Livorsi ${ }^{1,2^{*}}$, Michihiko Goto ${ }^{1,2}$, Margaret Carrel ${ }^{3}$, Makoto M. Jones ${ }^{4,5}$, Jennifer McDanel ${ }^{1,6}$, Rajeshwari Nair ${ }^{1,7}$, Bruce Alexander ${ }^{1}$, Brice Beck ${ }^{1}$, Kelly K. Richardson ${ }^{1}$ and Eli N. Perencevich ${ }^{1,7}$

\begin{abstract}
Objectives: We sought to define regional variations in fluoroquinolone non-susceptibility (FQ-NS) among bloodstream isolates of Escherichia coli across the Veterans Health Administration (VHA) in the United States.

Methods: We analyzed a retrospective cohort of patients managed at 136 VHA hospitals who had a blood culture positive for E.coli between 2003 and 2013. Hospitals were classified based on US Census Divisions, and regional variations in FQ-NS were analyzed.

Results: Twenty-four thousand five hundred twenty-three unique E.coli bloodstream infections (BSIs) were identified between 2003 and 2013. $53.9 \%$ of these were community-acquired, $30.7 \%$ were healthcare-associated, and $15.4 \%$ were hospital-onset BSIs. The proportion of E.coli BSIs with FQ-NS significantly varied across US Census Divisions $(p<0.001$ ). During 2003-2013, the proportion of E.coli BSIs with FQ-NS was highest in the West SouthCentral Division (32.7 \%) and lowest in the Mountain Division (20.0\%). Multivariable analysis showed that there were universal secular trends towards higher FQ-NS rates $(p<0.001)$ with significant variability of slopes across US Census Divisions $(p<0.001)$.
\end{abstract}

Conclusion: There has been a universal increase in FQ-NS among E.coli BSIs within VHA, but the rate of increase has significantly varied across Census Divisions. The reasons for this variability are unclear. These findings reinforce the importance of using local data to develop and update local antibiograms and antibiotic-prescribing guidelines.

Keywords: Fluoroquinolones, Antimicrobial resistance, Epidemiology, Escherichia coli,

\section{Introduction}

Fluoroquinolones are a synthetic class of antibiotics that have been used in clinical medicine since the 1970s. Fluoroquinolones have excellent oral bioavailability, provide a broad-spectrum of antibacterial activity, and are highly efficacious in the treatment of a variety of infections. However, the overuse of these agents has led to rising rates of FQ non-susceptibility (FQ-NS) [1-3].

\footnotetext{
* Correspondence: daniel-livorsi@uiowa.edu

${ }^{1}$ lowa City VA Health Care System, lowa City, IA, USA

${ }^{2}$ Division of Infectious Diseases, Department of Internal Medicine, University of lowa Carver College of Medicine, 200 Hawkins Drive, lowa City, IA 52242, USA

Full list of author information is available at the end of the article
}

Historically, fluoroquinolones have been reliably active against Escherichia coli, a common cause of urinary tract infections, bloodstream infections, and intra-abdominal infections. National antibiotic-prescribing guidelines still recommend the empiric use of fluoroquinolones for infections that commonly involve E.coli $[4,5]$, but given the rising prevalence of FQ-NS, the empiric use of fluoroquinolones may no longer be appropriate in some geographic regions [1-3].

In this study, we sought to define regional variations in FQ-NS among bloodstream isolates of E.coli in the Veterans Health Administration (VHA) over an 11-year period. Identifying temporal and regional differences in 
resistance patterns may inform development of national versus regional or local treatment guidelines.

\section{Methods}

We constructed a retrospective cohort of all patients within the VHA who had a blood culture positive for E.coli between January 1, 2003 and December 31, 2013. Data from 136 acute care hospitals in 48 US states contributed to the cohort. FQ-NS was defined as a nonsusceptible result to at least one FQ: ciprofloxacin, levofloxacin, and/or moxifloxacin. In line with guidelines from the Clinical and Laboratory Standards Institute, only the first isolate was included when the patient had more than one blood culture positive for E. coli in the same calendar year [6].

Bloodstream infections (BSIs) were defined as hospital-onset, healthcare-associated, or communityacquired. Hospital-onset BSIs were defined as an E.colipositive blood culture that was obtained after the patient had been hospitalized for $\geq 48 \mathrm{~h}$. Healthcare-associated BSIs were defined as an E.coli-positive blood culture obtained at the time of admission or $<48 \mathrm{~h}$ of admission if the patient met established criteria for healthcare exposure [7]. Community-acquired BSIs were defined as E.coli-positive blood cultures obtained at the time of admission or $<48 \mathrm{~h}$ of admission in patients who did not meet criteria for healthcare-associated infections.

Hospitals were regionalized based on US Census Divisions (https://www.census.gov/geo/reference/gtc/gtc_cen sus_divreg.html), and annual averages of FQ-NS were mapped. Monthly and annual counts of isolates with and without FQ-NS were measured as outcomes.

Poisson regression models were used to predict the number of isolates with FQ-NS while incorporating total number of isolates as an offset variable. The generalized estimating equations (GEE) method was used to account for grouping effect within Census Divisions. Residual plots were inspected to ensure the appropriateness of all models. An interaction term between time and region was included in the model, and region-specific incidence rate ratios (IRRs) by month and year were estimated to assess variability of slopes across divisions.

\section{Results}

Twenty-four thousand five hundred twenty-three unique E.coli (BSIs) were identified between 2003 and 2013 across 136 hospitals and 9 Census Divisions (Table 1). A majority of these BSIs were classified as communityacquired (53.9 \%); $30.7 \%$ were healthcare-associated and $15.4 \%$ were hospital-onset BSIs.

The prevalence of FQ-NS increased with healthcare exposure. The frequency of FQ-NS was $20.2 \%$ for community-acquired E.coli-BSIs, $37.0 \%$ for healthcare-
Table 1 Number of VHA hospitals and E.coli bloodstream isolates (BSIs) from each census region, 2003-2013

\begin{tabular}{lll}
\hline Census region & Number of hospitals & $\begin{array}{l}\text { Number of included } \\
\text { E. coli BSIs (\%) }\end{array}$ \\
\hline East North Central & 18 & $2799(11.4 \%)$ \\
East South Central & 11 & $2146(8.8 \%)$ \\
Middle Atlantic & 18 & $2544(10.4 \%)$ \\
Mountain & 13 & $2010(8.2 \%)$ \\
New England & 7 & $783(3.2 \%)$ \\
Pacific Central & 13 & $3253(13.3 \%)$ \\
South Atlantic & 26 & $5358(21.8 \%)$ \\
West North Central & 16 & $1896(7.7 \%)$ \\
West South Central & 14 & $3734(15.2 \%)$ \\
\hline
\end{tabular}

associated E.coli-BSIs, and $37.7 \%$ for hospital-onset E.coli-BSIs.

Throughout the VHA, the percentage of E.coli-BSIs with FQ-NS increased from $13.9 \%$ in 2003 to $31.3 \%$ in 2008 and $32.6 \%$ in 2013. Among E.coli strains demonstrating FQ-NS, the frequency of non-susceptibility to other antibiotic classes was as follows: $16.6 \%$ extendedspectrum cephalosporins, $34.4 \%$ aminoglycosides, and $0.3 \%$ carbapenems.

The proportion of E.coli-BSIs with FQ-NS significantly varied across Census Divisions (range: 20.0$32.7 \%$; $p<0.001$ ). During 2003-2013, the proportion of E.coli BSIs with FQ-NS was highest in the West South Central Division (32.7 \%) and lowest in the Mountain Division (20.0 \%, Fig. 1). In 2013 alone, the highest frequency of FQ-NS among E.coli-BSIs was seen in the East South Central Division (39.6 \%) and the lowest frequency of FQ-NS was seen in the Pacific Central Division (25.4 \%).

Regression analysis showed that there were universal temporal trends towards higher FQ-NS rates $(p<0.001)$ with significant variability of slopes across Census Divisions $(p<0.001$, Fig. 2). The annual mean rate of increase in FQ-NS was as follows: East North Central $0.41 \%$ /month; Mountain $0.86 \%$ /month; South Atlantic $0.54 \%$ /month; East South Central $0.51 \%$ /month; New England $0.44 \%$ /month; West North Central $0.89 \%$ /month; Middle Atlantic $0.45 \%$ /month; Pacific Central 0.19 \% /month; West South Central 0.40 \% /month.

\section{Discussion}

Our study has demonstrated a sustained increase in the frequency of FQ-NS among E.coli BSIs across VHA during 2003-2013. The rate of change has significantly varied across Census Divisions. The high prevalence of FQ-NS in some divisions could influence the empiric use of fluoroquinolones for infections thought to involve 


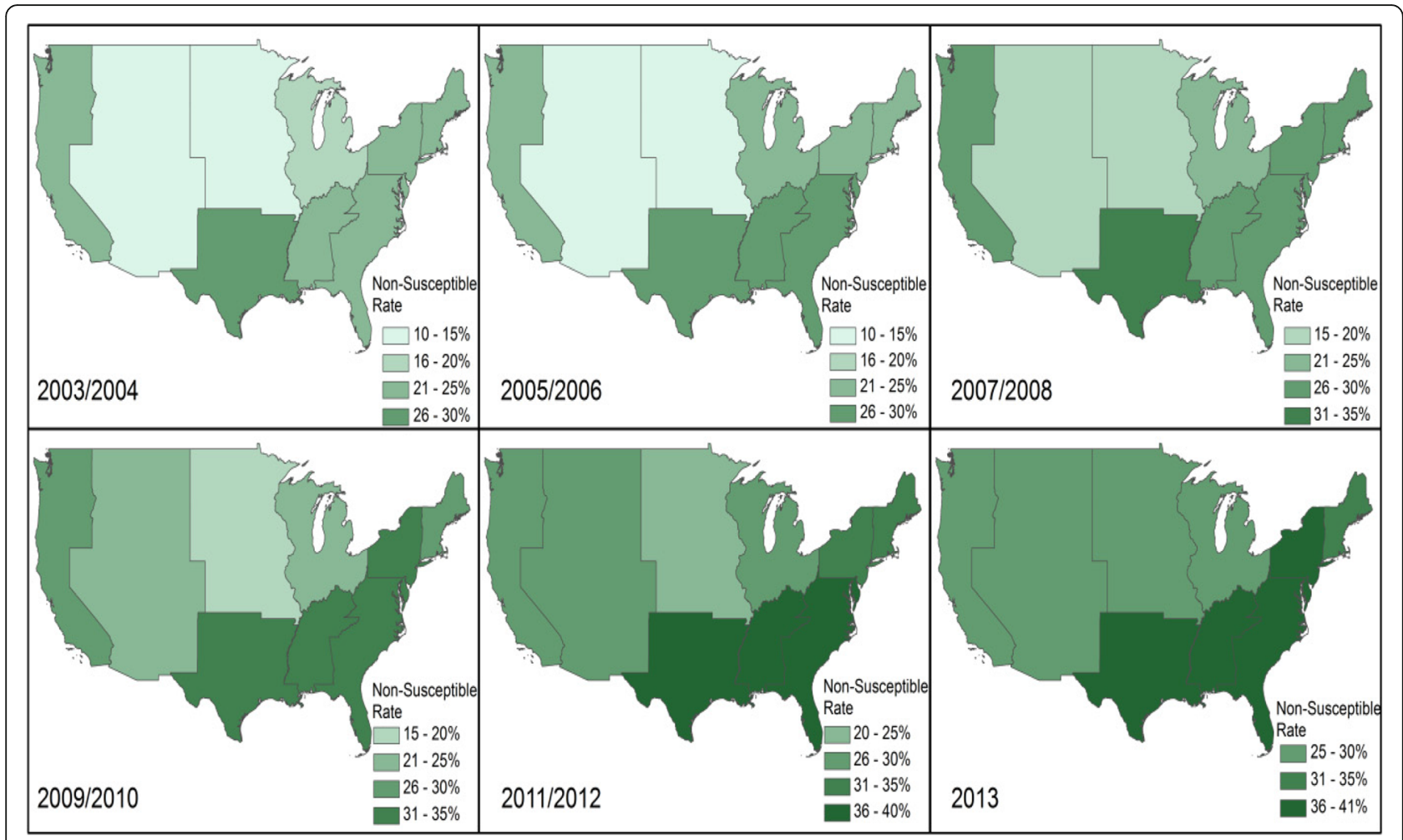

Fig. 1 Region-specific changes in FQ-NS among E.coli BSIs in the Veterans Health Administration, 2003-2013

E.coli and suggests that regional or even local guidelines might be preferred over national guidelines.

The clonal expansion of sequence type (ST) 131 E.coli is likely a major driver of this rising prevalence of fluoroquinolone resistance [8-11]. ST131 represents one of more than 1000 STs of E.coli defined by multilocus sequence typing (MLST). The clonal expansion of ST131 has been a global phenomenon that has not spared the VHA. In a 2011 analysis of E.coli clinical isolates from 24 VHA medical centers, ST131 accounted for $78 \%$ of fluoroquinolone-resistant isolates and $28 \%$ of all isolates [12]. The aminoglycoside and carbapenem susceptibility profiles of ST131 strains described in this 2011 study are similar to that of FQ-NS strains in our report. Studies outside of VHA have also found that ST131 accounts for 70-80 \% of fluoroquinoloneresistant E.coli infections [12-14]. A specific subclone, which represents the vast majority of fluoroquinoloneresistant ST131 isolates, first emerged around 2000 and has since expanded rapidly around the world [15].

The global dissemination of ST131 and its subclones is not well understood. Possible microbiologic contributors include the clone's enhanced transmissibility, its increased virulence, its resistance to multiple antibiotics, and its success at colonizing the human body [8].

In certain healthcare settings, there is a high colonization pressure with ST131, and this likely facilitates patient-to-patient transmission. Long-term care facilities may serve as reservoirs of this clone $[16,17]$. At 2 longterm care facilities in Minnesota, $24 \%$ of residents were colonized with ST131, and molecular analysis demonstrated evidence of intra-facility and inter-facility transmission [17]. Single-center studies at different acute care hospitals found that $50 \%$ of inpatients are carriers [18] and that $13 \%$ of stool samples sent to the microbiology laboratory grew E.coli ST131 [19].

Antibiotic-prescribing practices are probably also contributing to the spread of ST131. In a population-based cohort study, multivariable analysis showed that ST131 carriage was predicted by older age, healthcare exposure, and prior antibiotic use [13]. Specifically, prior use of fluoroquinolones, macrolides or extended-spectrum cephalosporins was predictive [13]. Other studies have found that prior fluoroquinolone-exposure is a risk factor for fluoroquinolone-resistant E.coli, but these studies did not describe the sequence type of the infecting strains of E.coli [2, 20, 21].

It's unclear why the frequency of FQ-NS significantly varied across Census Divisions within the VHA over time. These differences may reflect broad ecological trends beyond the VHA patient cohort. Alternately, specific infection control and antibiotic-prescribing practices within VHA may be influencing the spread of these strains of E.coli. Future research could examine 


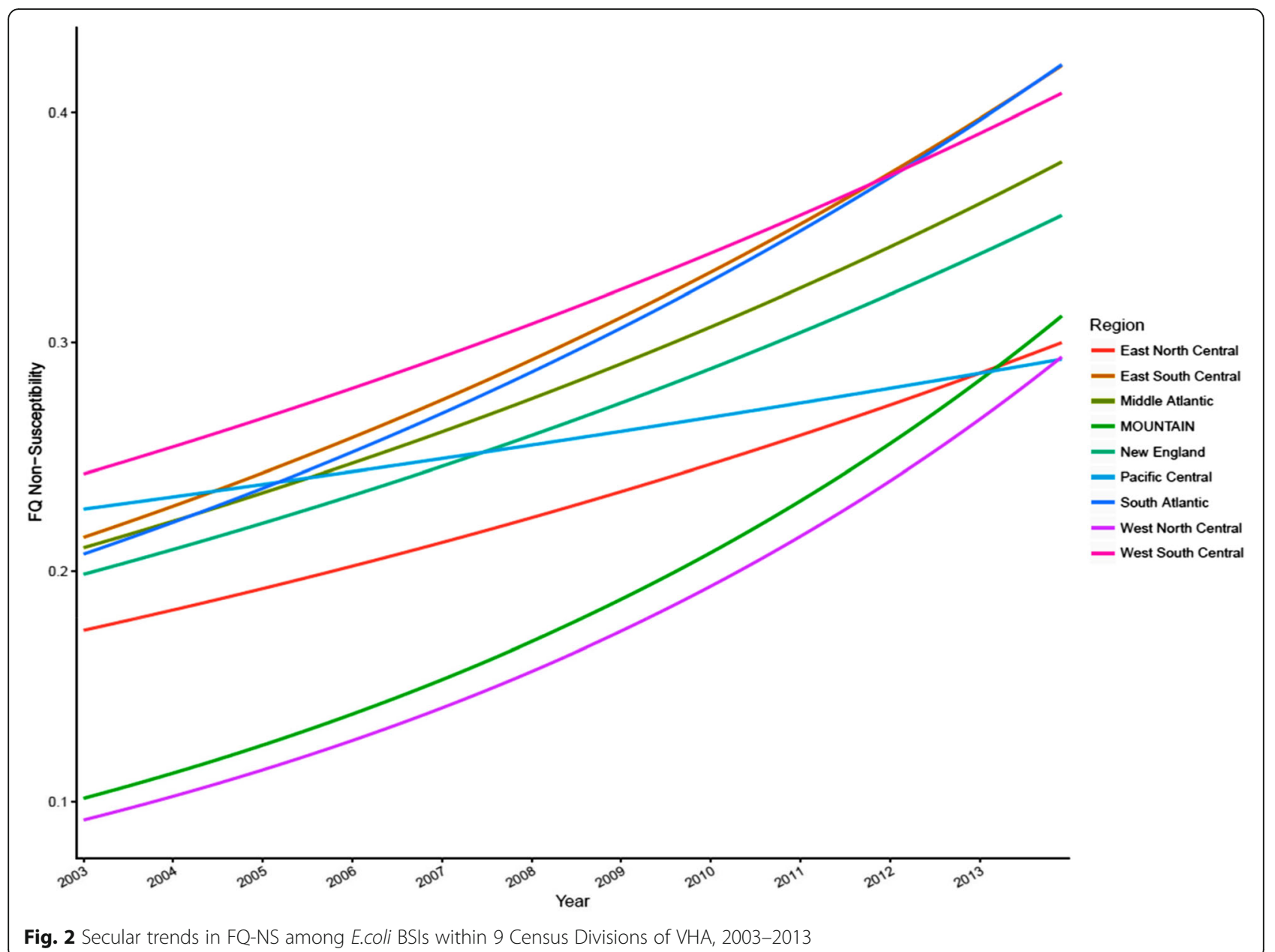

whether use of specific antibiotic agents varies across regions and, if so, whether the variations in antibiotic usage help explain the variability in FQ-NS.

There are several clinical implications to these changing resistance patterns among E.coli. Patients undergoing transrectal prostate biopsy typically receive fluoroquionolone prophylaxis. In one study, colonization with fluoroquinolone-resistant E.coli was the most important host characteristic associated with infection after transrectal prostate biopsy. Nearly two-thirds of these resistant strains were ST 131 [22]. A survey of Infectious Disease physicians found that there is a perceived increase in the incidence of infections after transrectal prostate biopsies [23]. Furthermore, rising rates of fluoroquinolone-resistant E.coli limit options for empiric antibiotic therapy. Among patients with E.coli bacteremia, inappropriate empiric antibiotic therapy was associated with worse outcomes in some [24, 25] but not all $[26,27]$ studies.

Our study has some limitations. First, the cohort included older patients who were predominantly male. As a result, the trends we observed may not be generalizable to other populations. Second, we have argued that ST131 is contributing to the changing epidemiology of E.coli, but we did not perform any microbiologic typing. A prior study, however, has demonstrated that ST 131 is prevalent within VHA [12].

\section{Conclusion}

In conclusion, there has been a universal increase in FQ-NS among E.coli BSIs within VHA, and the rate of increase has varied across Census Divisions. These findings reinforce the importance of using local data to develop and update local antibiograms and antibioticprescribing guidelines.

\section{Abbreviations}

BSI: Bloodstream infection; E.coli: Escherichia coli; FQ-NS: Fluoroquinolone non-susceptibility; GEE: Generalized estimating equations; VHA: Veterans Health Administration

\section{Acknowledgements}

Not applicable.

Funding

Not applicable. 


\section{Availability of data and material}

The data that support the findings of this study are available from the VA Informatics and Computing Infrastructure (VINCI) but restrictions apply to the availability of these data, which were used under license for the current study, and so are not publicly available. Data are however available from the authors upon reasonable request and with permission of $\mathrm{VINCl}$

\section{Authors' contributions}

Study design: DL. Data collection and analysis: MG, MJ, JM, RN, BA, BB, KR. Study supervision: EP. Manuscript writing: DL. Manuscript review: All authors. All authors read and approved the final manuscript.

\section{Competing interests}

The authors declare that they have no competing interests.

\section{Consent for publication}

Not applicable.

\section{Ethics approval}

The protocol was approved by the University of lowa Institutional Review Board and the local VHA Research and Development Committee.

\section{Author details}

${ }^{1}$ Iowa City VA Health Care System, lowa City, IA, USA. ${ }^{2}$ Division of Infectious Diseases, Department of Internal Medicine, University of lowa Carver College of Medicine, 200 Hawkins Drive, lowa City, IA 52242, USA. ${ }^{3}$ Department of Geographical and Sustainability Sciences, College of Liberal Arts and Sciences, University of lowa, lowa City, IA, USA. ${ }^{4}$ Salt Lake City VA Health Care System, Salt Lake City, UT, USA. ${ }^{5}$ University of Utah School of Medicine, Salt Lake City, UT, USA. ${ }^{6}$ Department of Epidemiology, College of Public Health, University of lowa, lowa City, IA, USA. 'Division of General Internal Medicine, Department of Internal Medicine, University of lowa Carver College of Medicine, lowa City, IA, USA.

Received: 19 July 2016 Accepted: 27 September 2016 Published online: 19 October 2016

\section{References}

1. Fasugba O, Gardner A, Mitchell BG, Mnatzaganian G. Ciprofloxacin resistance in community- and hospital-acquired Escherichia coli urinary tract infections: a systematic review and meta-analysis of observational studies. BMC Infect Dis. 2015;15:545.

2. Zervos MJ, Hershberger E, Nicolau DP, Ritchie DJ, Blackner LK, Coyle EA, Donnelly AJ, Eckel SF, Eng RH, Hiltz A, et al. Relationship between fluoroquinolone use and changes in susceptibility to fluoroquinolones of selected pathogens in 10 United States teaching hospitals, 1991-2000. Clin Infect Dis. 2003:37(12):1643-8.

3. Sanchez GV, Master RN, Karlowsky JA, Bordon JM. In vitro antimicrobial resistance of urinary Escherichia coli isolates among U.S. outpatients from 2000 to 2010. Antimicrob Agents Chemother. 2012;56(4):2181-3.

4. Solomkin JS, Mazuski JE, Bradley JS, Rodvold KA, Goldstein EJ, Baron EJ, O'Neill PJ, Chow AW, Dellinger EP, Eachempati SR, et al. Diagnosis and management of complicated intra-abdominal infection in adults and children: guidelines by the Surgical Infection Society and the Infectious Diseases Society of America. Clin Infect Dis. 2010;50(2):133-64.

5. Gupta K, Hooton TM, Naber KG, Wullt B, Colgan R, Miller LG, Moran GJ Nicolle LE, Raz R, Schaeffer AJ, et al. International clinical practice guidelines for the treatment of acute uncomplicated cystitis and pyelonephritis in women: A 2010 update by the Infectious Diseases Society of America and the European Society for Microbiology and Infectious Diseases. Clin Infect Dis. 2011;52(5):e103-20.

6. CLSI. Analysis and presentation of cumulative antimicrobial susceptibility test data. Wayne: Clinical and Laboratory Standards Institute; 2014

7. Friedman ND, Kaye KS, Stout JE, McGarry SA, Trivette SL, Briggs JP, Lamm W, Clark C, MacFarquhar J, Walton AL, et al. Health care-associated bloodstream infections in adults: a reason to change the accepted definition of communityacquired infections. Ann Intern Med. 2002;137(10):791-7.

8. Banerjee R, Johnson JR. A new clone sweeps clean: the enigmatic emergence of Escherichia coli sequence type 131. Antimicrob Agents Chemother. 2014;58(9):4997-5004
9. Rogers BA, Sidjabat HE, Paterson DL. Escherichia coli O25b-ST131: a pandemic, multiresistant, community-associated strain. J Antimicrob Chemother. $2011 ; 66(1): 1-14$.

10. Johnson JR, Johnston B, Clabots C, Kuskowski MA, Castanheira M. Escherichia coli sequence type ST131 as the major cause of serious multidrug-resistant E. coli infections in the United States. Clinlnfect Dis. 2010;51(3):286-94

11. Peirano G, Pitout JD. Molecular epidemiology of Escherichia coli producing CTX-M beta-lactamases: the worldwide emergence of clone ST131 O25:H4. Int J Antimicrob Agents. 2010;35(4):316-21.

12. Colpan A, Johnston B, Porter S, Clabots C, Anway R, Thao L, Kuskowski MA Tchesnokova V, Sokurenko EV, Johnson JR, et al. Escherichia coli sequence type 131 (ST131) subclone $\mathrm{H} 30$ as an emergent multidrug-resistant pathogen among US veterans. Clin Infect Dis. 2013;57(9):1256-65.

13. Banerjee $\mathrm{R}$, Johnston B, Lohse C, Porter SB, Clabots C, Johnson JR Escherichia coli sequence type 131 is a dominant, antimicrobial-resistant clonal group associated with healthcare and elderly hosts. Infect Control Hosp Epidemiol. 2013;34(4):361-9.

14. Croxall G, Hale J, Weston V, Manning G, Cheetham P, Achtman M, McNally A. Molecular epidemiology of extraintestinal pathogenic Escherichia coli isolates from a regional cohort of elderly patients highlights the prevalence of ST131 strains with increased antimicrobial resistance in both community and hospital care settings. J Antimicrob Chemother. 2011;66(11):2501-8.

15. Johnson JR, Tchesnokova V, Johnston B, Clabots C, Roberts PL, Billig M, Riddell K, Rogers P, Qin X, Butler-Wu S, et al. Abrupt emergence of a single dominant multidrug-resistant strain of Escherichia coli. J Infect Dis. 2013;207(6):919-28.

16. Maslow JN, Lee B, Lautenbach E. Fluoroquinolone-resistant Escherichia coli carriage in long-term care facility. Emerg Infect Dis. 2005;11(6):889-94.

17. Burgess MJ, Johnson JR, Porter SB, Johnston B, Clabots C, Lahr BD, Uhl JR, Banerjee R. Long-Term Care Facilities Are Reservoirs for AntimicrobialResistant Sequence Type 131 Escherichia coli. Open Forum Infectious Diseases. 2015;2(1):ofv011.

18. Han JH, Johnston B, Nachamkin I, Tolomeo P, Bilker WB, Mao X, Clabots C, Lautenbach $\mathrm{E}$, Johnson JR, Program CDCPE. Clinical and molecular epidemiology of Escherichia coli sequence type 131 among hospitalized patients colonized intestinally with fluoroquinolone-resistant E. coli. Antimicrob Agents Chemother. 2014:58(11):7003-6.

19. Mohamed M, Clabots C, Porter SB, Thuras P, Johnson JR. Isolation and Characterization of Escherichia coli Sequence Type 131 and Other Antimicrobial-Resistant Gram-Negative Bacilli from Clinical Stool Samples from Veterans. Antimicrob Agents Chemother. 2016;60(8):4638-45.

20. Loeb MB, Craven S, McGeer AJ, Simor AE, Bradley SF, Low DE, ArmstrongEvans M, Moss LA, Walter SD. Risk factors for resistance to antimicrobial agents among nursing home residents. Am J Epidemiol. 2003;157(1):40-7.

21. Carratala J, Fernandez-Sevilla A, Tubau F, Callis M, Gudiol F. Emergence of quinolone-resistant Escherichia coli bacteremia in neutropenic patients with cancer who have received prophylactic norfloxacin. Clin Infect Dis. 1995; 20(3):557-60.

22. Liss MA, Johnson JR, Porter SB, Johnston B, Clabots C, Gillis K, Nseyo U, Holden M, Sakamoto K, Fierer J. Clinical and microbiological determinants of infection after transrectal prostate biopsy. Clin Infect Dis. 2015;60(7):979-87.

23. Johnson JR, Polgreen PM, Beekmann SE. Transrectal prostate biopsyassociated prophylaxis and infectious complications: report of a query to the emerging infections network of the infectious diseases society of America. Open Forum Infectious Diseases. 2015;2(1):ofv002.

24. Peralta G, Sanchez MB, Garrido JC, De Benito I, Cano ME, Martinez-Martinez $\mathrm{L}$, Roiz MP. Impact of antibiotic resistance and of adequate empirical antibiotic treatment in the prognosis of patients with Escherichia coli bacteraemia. J Antimicrob Chemother. 2007:60(4):855-63.

25. Kuikka A, Sivonen A, Emelianova A, Valtonen W. Prognostic factors associated with improved outcome of Escherichia coli bacteremia in a Finnish university hospital. Eur J Clin Microbiol Infect Dis. 1997;16(2):125-34.

26. Thom KA, Schweizer ML, Osih RB, McGregor JC, Furuno JP, Perencevich EN, Harris AD. Impact of empiric antimicrobial therapy on outcomes in patients with Escherichia coli and Klebsiella pneumoniae bacteremia: a cohort study. BMC Infect Dis. 2008:8:116.

27. Kang Cl, Kim SH, Park WB, Lee KD, Kim HB, Kim EC, Oh MD, Choe KW. Bloodstream infections due to extended-spectrum beta-lactamaseproducing Escherichia coli and Klebsiella pneumoniae: risk factors for mortality and treatment outcome, with special emphasis on antimicrobial therapy. Antimicrob Agents Chemother. 2004;48(12):4574-81. 